\title{
Invariant vector means and complementability of Banach spaces in their second duals
}

\author{
RADOSŁAW ŁUKASIK(D)
}

\begin{abstract}
Let $X$ be a Banach space. Fix a torsion-free commutative and cancellative semigroup $S$ whose torsion-free rank is the same as the density of $X^{* *}$. We then show that $X$ is complemented in $X^{* *}$ if and only if there exists an invariant mean $M: \ell_{\infty}(S, X) \rightarrow X$. This improves upon previous results due to Bustos Domecq (J Math Anal Appl 275(2):512-520, 2002), Kania (J Math Anal Appl 445:797-802, 2017), Goucher and Kania (Studia Math 260:91-101, 2021).
\end{abstract}

Mathematics Subject Classification. Primary 43A07, Secondary 46B50.

Keywords. Commutative semigroup, Invariant mean, Vector-valued mean, Principle of local reflexivity, Banach space complemented in bidual, Commutative cancellative semigroup.

\section{Introduction}

Invariant means on amenable groups are an important tool in many parts of Mathematics, especially in Harmonic analysis (see [8,9]). For the basic properties of invariant means, we refer the reader to [8]. Invariant means and their generalizations for vector-valued functions also play an important role in the stability of functional equations and selections of set-valued functions (see $[1,5,6,16])$.

The space of all bounded functions from a set $S$ into a Banach space $X$ is denoted by $\ell_{\infty}(S, X)$. Let us recall the definition of an amenable semigroup (see [3]). 
Definition 1.1. A semigroup $(S,+)$ is called left [resp. right] amenable if and only if there exists a linear map $L: \ell_{\infty}(S, \mathbb{R}) \rightarrow \mathbb{R}$ such that

$$
\begin{aligned}
& \inf f(S) \leq L(f) \leq \sup f(S), f \in \ell_{\infty}(S, \mathbb{R}) \\
& L\left({ }_{a} f\right)=L(f), a \in S, f \in \ell_{\infty}(S, \mathbb{R}), \\
& {\left[L\left(f_{a}\right)=L(f), a \in S, f \in \ell_{\infty}(S, \mathbb{R})\right],}
\end{aligned}
$$

where

$$
\begin{aligned}
& { }_{a} f(x)=f(a+x), a, x \in S, f \in \ell_{\infty}(S, \mathbb{R}), \\
& {\left[f_{a}(x)=f(x+a), a, x \in S, f \in \ell_{\infty}(S, \mathbb{R})\right] .}
\end{aligned}
$$

If both left and right invariant means exist, then $S$ is called amenable.

Remark 1.2. In the above definition the first condition

$$
\inf f(S) \leq L(f) \leq \sup f(S)
$$

is equivalent to conditions $L\left(\mathbb{1}_{S}\right)=1$ and $|L(f)| \leq\|f\|:=\sup |f(S)|$.

It is known that every commutative semigroup is amenable (an easy consequence of the Markov-Kakutani fixed point theorem, see [15, Theorem 5.23]).

Certain generalizations of invariant means were investigated for vectorvalued functions in [5] and the existence thereof appears to be related to properties such as reflexivity.

Some generalized definition of an invariant mean has been used by many mathematicians as a folklore (e.g. by Pełczyński [14]). The explicit form of this definition can be found e.g. in the work of Ger [6].

Definition 1.3. Let $(S,+)$ be a left [right] amenable semigroup and $X$ be a Banach space. A linear map $M: \ell_{\infty}(S, X) \rightarrow X$ is called a left [right] $X$-valued invariant mean if

$$
\begin{aligned}
& \|M\| \leq 1, \\
& M\left(c \mathbb{1}_{S}\right)=c, c \in X, \\
& M\left({ }_{a} f\right)=M(f), a \in S, f \in \ell_{\infty}(S, X), \\
& {\left[M\left(f_{a}\right)=M(f), a \in S, f \in \ell_{\infty}(S, X),\right]}
\end{aligned}
$$

where

$$
\begin{aligned}
& { }_{a} f(x)=f(a+x), a, x \in S, f \in \ell_{\infty}(S, X), \\
& \quad\left[f_{a}(x)=f(x+a), a, x \in S, f \in \ell_{\infty}(S, X) .\right]
\end{aligned}
$$

If $M$ is a left and right invariant mean, then $M$ is called an $X$-valued invariant mean.

If in the above definition the norm of map $M$ is equal to at most $\lambda \geq 1$, then $M$ is called an $X$-valued invariant $\lambda$-mean. 
The existence of such invariant means for a fixed Banach space and for all amenable semigroups has been studied by Domecq [4, Theorem 1 and 2] and by the author in [12]. However, as observed by Lipecki in his Mathematical Review (MR1943762) of Bustos Domecq's paper, the proof of Theorem 2 contains a gap (a flawed choice of the semigroup, so we cannot use the Principle of Local Reflexivity). This gap was corrected by Kania [10].

Goucher and Kania [7] consider the following question (communicated privately to T. Kania by J.M.F. Castillo).

Suppose that a Banach space $X$ admits an invariant mean with respect to every/some commutative group. Must $X$ be complemented in $X^{* *}$ ?

They proved the following (see [7, Theorem A], [10, Theorem 1.2])

Theorem 1.4. Let $X$ be a Banach space and $\lambda \geq 1$. Then the following assertions are equivalent.

1. $X$ is complemented in $X^{* *}$ by a projection of norm at most $\lambda$;

2. for every amenable semigroup $S$ there exists an $X$-valued invariant $\lambda$ mean on $S$;

3. for every commutative semigroup $S$ there exists an $X$-valued invariant $\lambda$-mean on $S$;

4. for every free commutative group $G$ of rank $\left|X^{* *}\right|$ there exists an $X$-valued invariant $\lambda$-mean on $G$;

5. there exists an $X$-valued invariant $\lambda$-mean on the additive group of $X^{* *}$.

It is also demonstrated ( $[7$, Remark 1.1]) that there exists a commutative noncancellative semigroup $S$ (that could be chosen as large as one wishes) such that there exists an $X$-valued invariant mean on $S$.

In this paper we will prove that if $X$ is a Banach space and there exists an invariant $X$-valued mean on any arbitrary commutative cancellative semigroup $S$ of torsion-free rank dens $X^{* *}$, then $X$ is $\lambda$-complemented in $X^{* *}$.

\section{Preliminaries}

First we recall the definition of torsion-free rank (see [2]).

Definition 2.1. Let $S$ be a commutative cancellative semigroup. A set $A \subset S$ is independent if $\sum_{i=1}^{n} k_{i} a_{i}=\sum_{i=1}^{n} m_{i} a_{i}$ for any $n \in \mathbb{N}$ and $a_{i} \in A, k_{i}, m_{i} \in \mathbb{N}_{0}$, $i \in\{1, \ldots, n\}$ implies $k_{i}=m_{i}$ for $i \in\{1, \ldots, n\}$.

Let further $\mathcal{A}_{0}$ be the family of all independent sets $L$ in $S$ consisting only of elements whose order is infinite and such that $L$ is maximal with respect to these properties. The cardinal number of any set in $\mathcal{A}_{0}$ is called a torsion-free rank of $S$ and is denoted by $r_{0}(S)$ (all the sets in $\mathcal{A}_{0}$ have the same cardinal number). 
The density character of a Banach space $X$, denoted dens $X$, is the smallest cardinal $\kappa$ for which $X$ has a dense subset of cardinality $\kappa$.

Lemma 2.2. Let $X$ be an infinite-dimensional Banach space. Then

1. if $\mathcal{B}$ is a linearly independent subset of $X$ and $\mathbb{F}$ is a countable dense subfield of a scalar field of $X$, then $\left|\operatorname{span}_{\mathbb{F}} \mathcal{B}\right|=|\mathcal{B}|$;

2. for every closed subspace $Y$ of $X$ there exists a linearly independent subset $\mathcal{B}$ of $X$ such that $|\mathcal{B}|=\operatorname{dens} X, \overline{\operatorname{span}} \mathcal{B}=X, \overline{\operatorname{span}}(\mathcal{B} \cap Y)=Y$. Moreover, we can assume that the norm of each $x \in \mathcal{B}$ is equal to 1.

Proof. 1. We observe that

$$
\begin{aligned}
|\mathcal{B}| & \leq\left|\operatorname{span}_{\mathbb{F}} \mathcal{B}\right|=\left|\bigcup_{n \in \mathbb{N}}(\mathbb{F} \cdot \mathcal{B})^{n}\right| \leq|\mathbb{N}| \cdot \sup _{n \in \mathbb{N}}\left|(\mathbb{F} \cdot \mathcal{B})^{n}\right| \\
& =|\mathbb{N}| \cdot|\mathbb{F} \cdot \mathcal{B}|=|\mathbb{N}| \cdot|\mathbb{F}| \cdot|\mathcal{B}|=|\mathcal{B}| .
\end{aligned}
$$

2. Let $Y$ be a closed subspace of $X, D$ be a dense subset of $X$ such that $|D|=\operatorname{dens} X$ and $K$ be a dense subset of $Y$ such that $|K| \leq \operatorname{dens} X$. Let further

$$
\begin{aligned}
D_{1} & :=\left\{\frac{x}{\|x\|}: x \in K \backslash\{0\}\right\}, \\
D_{2} & :=\left\{\frac{x}{\|x\|}: x \in D \backslash\{0\}\right\} .
\end{aligned}
$$

Let further $\mathcal{B}_{1}$ be a maximal linearly independent subset of $D_{1}$ and $\mathcal{B}$ be a maximal linearly independent subset of $D_{1} \cup D_{2}$ such that $\mathcal{B}_{1} \subset \mathcal{B}$. We have $\overline{\operatorname{span}} \mathcal{B}_{1}=Y, \overline{\operatorname{span}} \mathcal{B}=X$ and $|\mathcal{B}| \leq\left|D_{1}\right|+\left|D_{2}\right|=\operatorname{dens} X$. Let $\mathbb{F}=\mathbb{Q}$ when $X$ is a real space or $\mathbb{F}=\mathbb{Q}(i)$ when $X$ is a complex space. Since $\operatorname{span}_{\mathbb{F}} \mathcal{B}$ is dense in $X,|\mathcal{B}|=\left|\operatorname{span}_{\mathbb{F}} \mathcal{B}\right| \geq \operatorname{dens} X$. We also note that the norm of each $x \in \mathcal{B}$ is equal 1 .

We will also require the version of the principle of local reflexivity due to Lindenstrauss and Rosenthal [11]. We denote by $\kappa: X \rightarrow X^{* *}$ the canonical embedding from a Banach space $X$ into the second dual.

Theorem 2.3. Let $X$ be a Banach space. Then for every finite-dimensional subspace $F \subset X^{* *}$ and each $\varepsilon \in(0,1]$ there exists a linear map $P_{F}^{\varepsilon}: F \rightarrow \kappa(X)$ such that

1. $(1-\varepsilon)\|x\| \leq\left\|P_{F}^{\varepsilon}(x)\right\| \leq(1+\varepsilon)\|x\|, x \in F$;

2. $P_{F}^{\varepsilon}(x)=x$ for $x \in F \cap \kappa(X)$.

It is a standard fact that subgroups and quotients of amenable groups are amenable. Using exactly the same ideas one can prove that if a Banach space admits an invariant mean with respect to a group, then it also does so with respect to subgroups and quotients of the group (see [12, Theorem 3.12] and [7, Lemma 2.3]). We would like to get a similar result for quotients of semigroups 
(subsemigroups of an amenable group need not be amenable) but first we must say something about normal semigroups and quotients of semigroups (see also $[17])$. Let $(S,+)$ be a semigroup, $G$ be a subsemigroup of $S$. Then $G$ is called a normal subsemigroup if $x+G=G+x$ for every $x \in S$. Of course in a commutative semigroup each subsemigroup is normal.

Let further $S$ be a semigroup and $G$ be a normal subsemigroup of $S$. We define the quotient semigroup $S / G:=S / \stackrel{G}{\sim}$, where $x \stackrel{G}{\sim} y$ iff $(x+G) \cap(y+G) \neq$ $\emptyset$. It is easy to notice that for any $g \in G$ the set $[g]_{G}$ is a neutral element of $S / G$. Moreover, if $G$ is a group, then $G$ is a neutral element of $S / G$.

Lemma 2.4. Let $S$ be an amenable semigroup and $G$ be a normal subsemigroup of $S$. If there exists an $X$-valued invariant $\lambda$-mean $M: \ell_{\infty}(S, X) \rightarrow X$, then there exists an $X$-valued invariant $\lambda$-mean $M: \ell_{\infty}(S / G, X) \rightarrow X$.

Proof. We define a map $M_{1}: \ell_{\infty}(S / G, X) \rightarrow X$ by the formula

$$
M_{1}(f):=M(\psi(f)), f \in \ell_{\infty}(S / G, X),
$$

where $\psi(f)(s)=f\left([s]_{\mathcal{\sim}}\right)$ for $s \in S$ and $f \in \ell_{\infty}(S / G, X)$. Since $\psi$ is linear, $\|\psi(f)\|=\|f\|$ and

$$
\begin{gathered}
\psi\left([t]_{\mathcal{\sim}} f\right)(s)={ }_{[t]_{G}} f\left([s]_{G}\right)=f\left([t+s]_{\mathcal{\sim}}\right)=\psi(f)(t+s)=\left({ }_{t} \psi(f)\right)(s), \\
\psi\left(f_{[t]_{\mathcal{\sim}}}\right)(s)=f_{[t]_{\mathcal{\sim}}}\left([s]_{\mathcal{\sim}}\right)=f\left([s+t]_{\mathcal{\sim}}\right)=\psi(f)(s+t)=\left(\psi(f)_{t}\right)(s),
\end{gathered}
$$

for all $s, t \in S, f \in \ell_{\infty}(S / G, X)$, then $M_{1}$ is an $X$-valued invariant $\lambda$-mean on $S / G$.

\section{Main results}

Throughout this section we fix an infinite-dimensional Banach space $X, \lambda \geq 1$. Let $\gamma$ be a cardinal number. We denote by $S_{\gamma}$ the commutative semigroup comprising all finite subsets of $\gamma$ endowed with the operation of taking the union of sets. It is easy to observe that $\left|S_{\gamma}\right|=\gamma$.

Theorem 3.1. Let $\gamma$ be an infinite cardinal number. If there exists an $X$-valued invariant $\lambda$-mean $M: \ell_{\infty}\left(S_{\gamma}, X\right) \rightarrow X$, then for every subspace $E$ of $X^{* *}$ such that dens $E=\gamma$ there exists a linear map $P: E \rightarrow X$ such that $\|P\| \leq \lambda$ and $P(x)=x$ for $x \in \kappa(X) \cap E$.

Proof. Let $\mathbb{K}$ be a scalar field of $X$. In view of Lemma 2.2 there exists a linearly independent subset $\mathcal{B}$ of $E$ such that $\overline{\operatorname{span}} \mathcal{B}=E, \overline{\operatorname{span}}(\mathcal{B} \cap \kappa(X))=\kappa(X) \cap E$, $|\mathcal{B}|=\operatorname{dens} E=\gamma$. Let $T: \gamma \rightarrow \mathcal{B}$ be a bijection and $M: \ell_{\infty}\left(S_{\gamma}, X\right) \rightarrow X$ be an $X$-valued invariant $\lambda$-mean .

For $A \in S_{\gamma}$ we define $\varepsilon_{A}:=\frac{1}{|A|+1}$ and $P_{\operatorname{span} T(A)}^{\varepsilon_{A}}$ is a fixed linear operator satisfying the conditions of Theorem 2.3. 
We define the map $P: E \rightarrow X$ in the following way (on the dense subspace $\operatorname{span} \mathcal{B}$, the map is simply continuously extended to the closure): for $x \in \operatorname{span} \mathcal{B}$ we put $P(x):=M\left(\phi_{x}\right)$, where

$$
\phi_{x}(A):=\left\{\begin{array}{ll}
P_{\mathrm{span} T(A)}^{\varepsilon_{A}}(x), & x \in \operatorname{span} T(A) \\
0, & x \notin \operatorname{span} T(A)
\end{array}, A \in S_{\gamma}\right.
$$

when $x \in \mathcal{B}$ and

$$
\phi_{x}(A):=\sum_{i=1}^{n} \lambda_{i} \phi_{x_{i}}(A), A \in S_{\gamma}
$$

when $x=\sum_{i=1}^{n} \lambda_{i} x_{i}, \lambda_{1}, \ldots, \lambda_{n} \in \mathbb{K}, x_{1}, \ldots, x_{n} \in \mathcal{B}$.

For $x, y \in \operatorname{span} \mathcal{B}$ and $\alpha \in \mathbb{K}$ we notice that $\phi_{\alpha x+y}=\alpha \phi_{x}+\phi_{y}$. Thus

$$
P(\alpha x+y)=M\left(\phi_{\alpha x+y}\right)=\alpha M\left(\phi_{x}\right)+M\left(\phi_{y}\right)=\alpha P(x)+P(y),
$$

so $P$ is linear on $\operatorname{span} \mathcal{B}$.

Let $x=\sum_{i=1}^{n} \lambda_{i} x_{i}$ for some $\lambda_{1}, \ldots, \lambda_{n} \in \mathbb{K}, x_{1}, \ldots, x_{n} \in \mathcal{B}$. Let further $A_{0} \in S_{\gamma}$ be such that $x_{1}, \ldots x_{n} \in T\left(A_{0}\right)$.

We observe that

$$
\begin{aligned}
\|P(x)\| & =\left\|M\left(\phi_{x}\right)\right\|=\left\|M\left(\phi_{x}\left(\cdot \cup A_{0}\right)\right)\right\| \leq \lambda \sup _{A \in S_{\gamma}}\left\|\phi_{x}\left(A \cup A_{0}\right)\right\| \\
& =\lambda \sup _{A \in S_{\gamma}}\left\|\sum_{i=1}^{n} \lambda_{i} \phi_{x_{i}}\left(A \cup A_{0}\right)\right\|=\lambda \sup _{A \in S_{\gamma}}\left\|\sum_{i=1}^{n} \lambda_{i} P_{\operatorname{span}\left(A \cup A_{0}\right)}^{\varepsilon_{A \cup A_{0}}}\left(x_{i}\right)\right\| \\
& =\lambda \sup _{A \in S_{\gamma}}\left\|P_{\operatorname{span} T\left(A \cup A_{0}\right)}^{\varepsilon_{A \cup A_{0}}}\left(\sum_{i=1}^{n} \lambda_{i} x_{i}\right)\right\|=\lambda \sup _{A \in S_{\gamma}} \| P_{\operatorname{span} T\left(A \cup A_{0}\right)}^{\varepsilon_{A \cup A_{0}}(x) \|} \\
& \leq \lambda \sup _{A \in S_{\gamma}}\left(1+\varepsilon_{A \cup A_{0}}\right)\|x\| \leq \lambda\left(1+\frac{1}{1+\left|A_{0}\right|}\right)\|x\| .
\end{aligned}
$$

Since $A_{0}$ is arbitrary, we get $\|P(x)\| \leq \lambda\|x\|$.

Moreover, if $x \in \kappa(X)$, then from the properties of $\mathcal{B}$ we get $x_{1}, \ldots, x_{n} \in \kappa(X)$ and

$$
\begin{aligned}
\phi_{x}\left(A \cup A_{0}\right) & =\sum_{i=1}^{n} \lambda_{i} \phi_{x_{i}}\left(A \cup A_{0}\right)=\sum_{i=1}^{n} \lambda_{i} P_{\operatorname{span} T\left(A \cup A_{0}\right)}^{\varepsilon_{A \cup A_{0}}}\left(x_{i}\right) \\
& =\sum_{i=1}^{n} \lambda_{i} x_{i}=x, A \in S_{\gamma} .
\end{aligned}
$$

Hence

$$
P(x)=M\left(\phi_{x}\right)=M\left(\phi_{x}\left(\cdot \cup A_{0}\right)\right)=x .
$$


Theorem 3.2. Let $S$ be a commutative cancellative semigroup of torsion-free rank $\delta, \gamma=\max (\delta, \omega)$. If there exists an $X$-valued invariant $\lambda$-mean $M_{S}: \ell_{\infty}(S, X) \rightarrow X$, then there exists an $X$-valued invariant $\lambda$-mean $M: \ell_{\infty}\left(S_{\gamma}, X\right) \rightarrow X$.

Proof. First we observe that we can assume that $S$ contains only elements of infinite order. Indeed the set $G$ of all elements of finite order is a group and a torsion-free rank of $S / G$ is equal to $\gamma$. In view of Lemma 2.4 there exists an $X$-valued invariant $\lambda$-mean on $S / G$.

Let $A \subset S$ be a maximal linearly independent set. Hence $|A|=\delta$.

- First assume that $|A|=\gamma$ and let $A=\left\{x_{\alpha}: \alpha<\gamma\right\}$. For each $x \in S$ we define a set

$$
D_{x}:=\left\{x_{1}, \ldots, x_{n} \in A: \exists_{k, k_{1}, \ldots, k_{n} \in \mathbb{N}} \exists_{I \subset\{1, \ldots, n\}} k x+\sum_{i \in I} k_{i} x_{i}=\sum_{i \notin I} k_{i} x_{i}\right\} .
$$

First, we show that the above set is well-defined. If there exist $k, m \in \mathbb{N}$, $k_{1}, \ldots k_{n}, m_{1}, \ldots m_{n} \in \mathbb{N} \cup\{0\}, x_{1}, \ldots, x_{n} \in A$, and $I, J \subset\{1, \ldots, n\}$ such that $k_{i} \neq 0$ for $i \in I, m_{i} \neq 0$ for $i \in J$ and

$$
\begin{aligned}
& k x+\sum_{i \in I} k_{i} x_{i}=\sum_{i \notin I} k_{i} x_{i}, \\
& m x+\sum_{i \in J} m_{i} x_{i}=\sum_{i \notin J} m_{i} x_{i},
\end{aligned}
$$

then

$$
m k x+\sum_{i \in I} m k_{i} x_{i}+\sum_{i \notin J} k m_{i} x_{i}=k m x+\sum_{i \in J} k m_{i} x_{i}+\sum_{i \notin I} m k_{i} x_{i},
$$

whence

$$
\sum_{i \in I} m k_{i} x_{i}+\sum_{i \notin J} k m_{i} x_{i}=\sum_{i \in J} k m_{i} x_{i}+\sum_{i \notin I} m k_{i} x_{i}
$$

so

$$
\begin{aligned}
& \sum_{i \in I \cap J} m k_{i} x_{i}+\sum_{i \in I \backslash J}\left(m k_{i}+k m_{i}\right) x_{i}+\sum_{i \notin I \cup J} k m_{i} x_{i} \\
& =\sum_{i \in I \cap J} k m_{i} x_{i}+\sum_{i \in J \backslash I}\left(k m_{i}+m k_{i}\right) x_{i}+\sum_{i \notin I \cup J} m k_{i} x_{i} .
\end{aligned}
$$

As $A$ is linearly independent, we have $I \backslash J=J \backslash I=\emptyset$, which means that $I=J$. Thus we get that $k m_{i}=m k_{i}$ for $i \in\{1, \ldots, n\}$, so $D_{x}$ is well-defined.

We define a map $\varphi: \ell_{\infty}\left(S_{\gamma}, X\right) \rightarrow \ell_{\infty}(S, X)$ by the formula

$$
\varphi(f)(x):=f\left(\left\{\alpha<\gamma: x_{\alpha} \in D_{x}\right\}\right), x \in S, f \in \ell_{\infty}\left(S_{\gamma}, X\right) .
$$

It is easy to observe that $\varphi$ is linear, $\|\varphi(f)\|=\|f\|$ for $f \in \ell_{\infty}\left(S_{\gamma}, X\right)$ and $\varphi\left(c \mathbb{1}_{S_{\gamma}}\right)=c \mathbb{1}_{S}$ for $c \in X$. 
Let $M_{S}: \ell_{\infty}(S, X) \rightarrow X$ be an $X$-valued invariant $\lambda$-mean. We define $M: \ell_{\infty}\left(S_{\gamma}, X\right) \rightarrow X$ by the formula

$$
M(f):=M_{S}(\varphi(f)), f \in \ell_{\infty}\left(S_{\gamma}, X\right) .
$$

From the properties of $\varphi$ we obtain that $M$ is linear, $M\left(c \mathbb{1}_{S_{\gamma}}\right)=c$ for $c \in X$, and $\|M\| \leq\left\|M_{S}\right\| \leq \lambda$.

Now we show that $M$ is invariant. Let $f \in \ell_{\infty}\left(S_{\gamma}, X\right)$ and $A \in S_{\gamma}$. Since $A=\left\{\alpha_{1}, \ldots, \alpha_{n}\right\}$, from the invariance on each singleton $\left\{\alpha_{i}\right\}$ we obtain

$$
\begin{aligned}
& M\left({ }_{A} f\right)=M\left(\left\{\alpha_{1}\right\}\left(\left\{\alpha_{2}, \ldots, \alpha_{n}\right\} f\right)\right)=M\left(\left\{\alpha_{2}, \ldots, \alpha_{n}\right\} f\right)=\ldots \\
& =M\left(\left\{\alpha_{n}\right\} f\right)=M(f), f \in \ell_{\infty}\left(S_{\gamma}, X\right) .
\end{aligned}
$$

Hence we need to prove the invariance on each singleton, so we can assume that $A=\{\beta\}$ for some $\beta<\gamma$. Let $Z:=\left\{x \in S: x_{\beta} \notin D_{x+x_{\beta}}\right\}$. We show that

$$
Z \cap\left(m x_{\beta}+Z\right)=\emptyset, m \in \mathbb{N} .
$$

Suppose that $x \in Z \cap\left(m x_{\beta}+Z\right)$ for some $m \in \mathbb{N}$. Then there exists $y \in Z$ such that $x=m x_{\beta}+y$. Hence $x_{\beta} \notin D_{y+x_{\beta}} \cup D_{y+(m+1) x_{\beta}}$ but on the other hand, if $x_{\beta} \notin D_{y+x_{\beta}}$, then $x_{\beta} \in D_{y+(m+1) x_{\beta}}$, so we have a contradiction.

Since $S$ is cancellative, from (3.1) we obtain that

$$
\left(n x_{\beta}+Z\right) \cap\left(m x_{\beta}+Z\right)=\emptyset, m, n \in \mathbb{N}_{0}, m>n .
$$

Let $g \in \ell_{\infty}(S, X)$ be such that $g(x)=0$ for $x \in S \backslash Z$. From (3.2) we get

$$
\begin{aligned}
n\left\|M_{S}(g)\right\| & =\left\|\sum_{i=1}^{n} M_{S}\left(i x_{\beta} g\right)\right\|=\left\|M_{S}\left(\sum_{i=1}^{n} i x_{\beta} g\right)\right\| \\
& \leq \lambda\left\|\sum_{i=1}^{n} i x_{\beta} g\right\| \leq \lambda\|g\|, n \in \mathbb{N},
\end{aligned}
$$

so $M_{S}(g)=0$.

For each $y \in S$ we have

- if $x_{\beta} \notin D_{y}$, then $D_{y+x_{\beta}}=D_{y} \cup\left\{x_{\beta}\right\}$, so

$$
\begin{aligned}
& \varphi(\{\beta\} f)(y)=f\left(\left\{\alpha<\gamma: x_{\alpha} \in D_{y}\right\} \cup\{\beta\}\right) \\
& =f\left(\left\{\alpha<\gamma: x_{\alpha} \in D_{y+x_{\beta}}\right\}\right)=\left(_{x_{\beta}} \varphi(f)\right)(y) ;
\end{aligned}
$$

- if $x_{\beta} \in D_{y}$ and $x_{\beta} \in D_{y+x_{\beta}}$, then $D_{y+x_{\beta}}=D_{y}$, so

$$
\begin{aligned}
& \varphi(\{\beta\})(y)=f\left(\left\{\alpha<\gamma: x_{\alpha} \in D_{y}\right\} \cup\{\beta\}\right)=f\left(\left\{\alpha<\gamma: x_{\alpha} \in D_{y}\right\}\right) \\
& =f\left(\left\{\alpha<\gamma: x_{\alpha} \in D_{y+x_{\beta}}\right\}\right)=\left(_{x_{\beta}} \varphi(f)\right)(y) ;
\end{aligned}
$$

- if $x_{\beta} \notin D_{y+x_{\beta}}$, then $y \in Z$. 
Hence

$$
\left(\varphi(\{\beta\} f)-_{x_{\beta}} \varphi(f)\right)(y)=0, y \in S \backslash Z,
$$

SO

$$
M\left({ }_{\{\beta\}} f\right)=M_{S}\left(\varphi\left({ }_{\{\beta\}} f\right)=M_{S}\left(x_{\beta} \varphi(f)\right)=M_{S}(\varphi(f))=M(f) .\right.
$$

- Now assume that $|A|<\gamma$. Hence $\gamma=\omega$. Let $N=|A|, A=\left\{x_{1}, \ldots, x_{N}\right\}$. Since $S$ can be embedded in a group, for each $x \in S$ there exist $k(x) \in \mathbb{N}$, $k_{1}(x), \ldots, k_{N}(x) \in \mathbb{Z}$ such that $k(x) x=\sum_{i=1}^{N} k_{i}(x) x_{i}$. We define a map $\varphi: \ell_{\infty}\left(S_{\omega}, X\right) \rightarrow \ell_{\infty}(S, X)$ by the formula

$$
\varphi(f)(x):=f\left(\left\{\alpha \in \omega: \alpha k(x) \leq\left|k_{1}(x)\right|\right\}\right), x \in S, f \in \ell_{\infty}\left(S_{\omega}, X\right) .
$$

It is easy to observe that $\varphi$ is linear, $\|\varphi(f)\| \leq\|f\|$ for $f \in \ell_{\infty}\left(S_{\omega}, X\right)$ and $\varphi\left(c \mathbb{1}_{S_{\omega}}\right)=c \mathbb{1}_{S}$ for $c \in X$.

Let $M_{S}: \ell_{\infty}(S, X) \rightarrow X$ be an $X$-valued invariant $\lambda$-mean. We define $M: \ell_{\infty}\left(S_{\omega}, X\right) \rightarrow X$ by the formula

$$
M(f):=M_{S}(\varphi(f)), f \in \ell_{\infty}\left(S_{\omega}, X\right) .
$$

From the properties of $\varphi$ we obtain that $M$ is linear, $M\left(c \mathbb{1}_{S_{\gamma}}\right)=c$ for $c \in X$, and $\|M\| \leq \lambda$.

Now we show that $M$ is invariant. Let $f \in \ell_{\infty}\left(S_{\omega}, X\right)$ and $A \in S_{\omega}$. Similarly as in the previous case we need only to prove the invariance on each singleton, so we can assume that $A=\{\beta\}$ for some $\beta \in \omega$. Let

$$
Z:=\left\{x \in S:\left|k_{1}(x)\right|<\beta k(x)\right\} .
$$

We show that

$$
Z \cap\left(2 m \beta x_{1}+Z\right)=\emptyset, m \in \mathbb{N} .
$$

Suppose that $x \in Z \cap\left(m x_{\beta}+Z\right)$ for some $m \in \mathbb{N}$. Then there exists $y \in Z$ such that $x=2 m \beta x_{1}+y$. Hence

$$
k(y)\left[y+2 m \beta x_{1}\right]=\left[k_{1}(y)+2 m k(y) \beta\right] x_{1}+\sum_{i=2}^{N} k_{i}(y) x_{i},
$$

which gives us

$$
\beta k(y)>k_{1}(y)+2 m \beta k(y)>-\beta k(y)+2 \beta k(y)=\beta k(y),
$$

so we have a contradiction.

Since $S$ is cancellative, from (3.3) we obtain that

$$
\left(2 n \beta x_{1}+Z\right) \cap\left(2 m \beta x_{1}+Z\right)=\emptyset, m, n \in \mathbb{N}_{0}, m>n .
$$


Now observe that for $x \in S \backslash Z$ we have

$$
\begin{aligned}
\varphi\left(f_{\{\beta\}}(x)\right) & =f_{\{\beta\}}\left(\left\{\alpha \in \omega: \alpha k(x) \leq\left|k_{1}(x)\right|\right\}\right) \\
& =f\left(\left\{\alpha \in \omega: \alpha k(x) \leq\left|k_{1}(x)\right|\right\} \cup\{\beta\}\right) \\
& =f\left(\left\{\alpha \in \omega: \alpha k(x) \leq\left|k_{1}(x)\right|\right\}\right)=\varphi(f(x)),
\end{aligned}
$$

so from (3.4) we obtain that

$$
\begin{aligned}
& n\left\|M\left(f-f_{\{\beta\}}\right)\right\|=\left\|n M_{S}\left(\varphi(f)-\varphi\left(f_{\{\beta\}}\right)\right)\right\| \\
& =\left\|\sum_{i=1}^{n} M_{S}\left(\left(\varphi(f)-\varphi\left(f_{\{\beta\}}\right)\right)_{2 i \beta x_{1}}\right)\right\| \\
& =\left\|M_{S}\left(\sum_{i=1}^{n}\left(\varphi(f)-\varphi\left(f_{\{\beta\}}\right)\right)_{2 i \beta x_{1}}\right)\right\| \leq \lambda\left\|\varphi(f)-\varphi\left(f_{\{\beta\}}\right)\right\|
\end{aligned}
$$

for every $n \in \mathbb{N}$, which means that $M\left(f_{\{\beta\}}\right)=M(f)$.

Using Theorems 1.4, 3.1 and 3.2 we obtain the following

Corollary 3.3. The following assertions are equivalent:

1. $X$ is complemented in $X^{* *}$ by a projection of norm at most $\lambda$;

2. for every amenable semigroup $S$ there exists an $X$-valued invariant $\lambda$ mean on $S$;

3. for any cancellative semigroup $S$ of torsion-free rank $\delta$, dens $X^{* *}=\max (\delta, \omega)$, there exists an $X$-valued invariant $\lambda$-mean on $S$.

The following example shows that in general in the third assertion of the previous corollary the torsion-free rank of semigroup $S$ cannot be less than the density of $X$.

Example 3.4. Let $\Gamma$ be an uncountable set such that $|\Gamma|$ is a regular cardinal number. We define the set

$$
X:=\left\{f \in \ell_{\infty}(\Gamma):|\{\alpha \in \Gamma: f(\alpha) \neq 0\}|<|\Gamma|\right\} .
$$

It is easy too see that $X$ is a Banach space. Since $\mathbb{1}_{\{\alpha\}} \in X$ for $\alpha \in \Gamma$, dens $X=|\Gamma|$.

Let $S$ be an amenable semigroup, $|S|<\operatorname{dens} X$ and $L: \ell_{\infty}(S, \mathbb{R}) \rightarrow \mathbb{R}$ be an invariant mean. We define $M: \ell_{\infty}(S, X) \rightarrow X$ by the formula

$$
M(g)(\alpha):=L(g(\cdot)(\alpha)), g \in \ell_{\infty}(S, X), \alpha \in \Gamma .
$$

First, we observe that

$$
\begin{aligned}
\{\alpha \in \Gamma: M(g)(\alpha) \neq 0\} & =\{\alpha \in \Gamma: L(g(\cdot)(\alpha)) \neq 0\} \\
& \subset \bigcup_{s \in S}\{\alpha \in \Gamma: g(s)(\alpha) \neq 0\}
\end{aligned}
$$


and since $|\Gamma|$ is regular, we have

$$
|\{\alpha \in \Gamma: M(g)(\alpha) \neq 0\}| \leq|S| \cdot \sup _{s \in S}|\{\alpha \in \Gamma: g(s)(\alpha) \neq 0\}|<|\Gamma|,
$$

so $M$ is well-defined.

It is easy to see that $M$ is linear. We have also

$$
\begin{aligned}
\|M(g)\| & =\sup _{\alpha \in \Gamma}|M(g)(\alpha)|=\sup _{\alpha \in \Gamma}|L(g(\cdot)(\alpha))| \\
& \leq \sup _{\alpha \in \Gamma} \sup _{s \in S}|g(s)(\alpha)|=\sup _{s \in S}\|g(s)\|=\|g\|, g \in \ell_{\infty}(S, X),
\end{aligned}
$$

and

$$
M\left(c \mathbb{1}_{S}\right)(\alpha)=L\left(c(\alpha) \mathbb{1}_{S}\right)=c(\alpha), c \in X, \alpha \in \Gamma .
$$

Finally, we observe that

$$
\begin{aligned}
M\left({ }_{a} g\right)(\alpha) & =L(g(a+\cdot)(\alpha))=L(g(\cdot)(\alpha)) \\
& =M(g)(\alpha), g \in \ell_{\infty}(S, X), a \in S, \alpha \in \Gamma,
\end{aligned}
$$

so $M$ is an $X$-valued invariant mean.

In the paper of Pełczyński and Sudakov [13, Theorem 1] it is shown that $X$ isn't complemented in its bidual.

\section{Acknowledgements}

We wish to thank Tomasz Kania for insightful e-mail exchanges.

Open Access. This article is licensed under a Creative Commons Attribution 4.0 International License, which permits use, sharing, adaptation, distribution and reproduction in any medium or format, as long as you give appropriate credit to the original author(s) and the source, provide a link to the Creative Commons licence, and indicate if changes were made. The images or other third party material in this article are included in the article's Creative Commons licence, unless indicated otherwise in a credit line to the material. If material is not included in the article's Creative Commons licence and your intended use is not permitted by statutory regulation or exceeds the permitted use, you will need to obtain permission directly from the copyright holder. To view a copy of this licence, visit http:// creativecommons.org/licenses/by/4.0/.

Publisher's Note Springer Nature remains neutral with regard to jurisdictional claims in published maps and institutional affiliations.

\section{References}

[1] Badora, R., Ger, R., Páles, Zs.: Additive selections and the stability of the Cauchy functional equation, ANZIAM J. 44 (2003), 323-337

[2] Cegarra, A.M., Petrich, M.: The rank of a commutative cancellative semigroup. Acta Math. Hungar. 112(1-2), 71-75 (2005)

[3] Day, M.M.: Amenable semigroups. Illinois J. Math. 1(4), 509-544 (1957) 
[4] Bustos Domecq, H.: Vector-valued invariant means revisited. J. Math. Anal. Appl. 275(2), 512-520 (2002)

[5] Gajda, Z.: Invariant means and representations of semigroups in the theory of functional equations, Prace Naukowe Uniwersytetu Śląskiego, Katowice (1992)

[6] Ger, R.: The singular case in the stability behavior of linear mappings. Grazer Math. Ber. 316, 59-70 (1992)

[7] Goucher, A.P., Kania, T.: Invariant means on abelian groups capture complementability of Banach spaces in their second duals. Studia Math. 260, 91-101 (2021)

[8] Greenleaf, F.P.: Invariant means on topological groups and their applications, Van Nostrand Mathematical Studies, No. 16, Van Nostrand Reinhold Co., New York-Toronto, Ont.-London (1969)

[9] Hewitt, E., Ross, K.: Abstract Harmonic Analysis, vol. 1. Academic Press, New York (1962)

[10] Kania, T.: Vector-valued invariant means revisited once again. J. Math. Anal. Appl. 445, 797-802 (2017)

[11] Lindenstrauss, J., Rosenthal, H.P.: The $\mathcal{L}_{p}$ spaces. Israel J. Math. 7, 325-349 (1969)

[12] Eukasik, R.: Invariant means on Banach spaces. Ann. Math. Sil. 31, 127-140 (2017)

[13] Pełczyński, A., Sudakov, V.N.: Remark on non-complemented subspaces of the spaces $m(S)$. Colloquium Math. 9(1), 85-88 (1962)

[14] Pełczyński, A.: Linear extensions, linear averagings, and their applications to linear topological classification of spaces of continuous functions. Rozprawy Mat. 58, 92 (1968)

[15] Rudin, W.: Functional Analysis. McGraw-Hill, New York (1991)

[16] Székelyhidi, L.: A note on Hyers theorem. C. R. Math. Rep. Acad. Sci. Canada 8, 127-129 (1986)

[17] Xing, R., Wei, C., Liu, S.: Quotient semigroups and extension semigroups. Proc. Indian Acad. Sci. (Math. Sci.) 122, 339-350 (2012)

Radosław Łukasik

Institute of Mathematics

University of Silesia

ul. Bankowa 14

40-007 Katowice

Poland

e-mail: radoslaw.lukasik@us.edu.pl

Received: August 10, 2021

Revised: December 30, 2021

Accepted: January 1, 2022 